\title{
Décubitus ventral dans les cas d'insuffisance respiratoire hypoxémique liés à la COVID-19
}

\author{
Kevin Venus MD, Laveena Munshi MD MSc, Michael Fralick MD PhD
}

Citation : CMAJ 2020 November 23;192:E1531-7. doi : 10.1503/cmaj.201201-f; diffusion hâtive le 11 novembre 2020

Voir la version anglaise de l'article ici : www.cmaj.ca/lookup/doi/10.1503/cmaj.201201

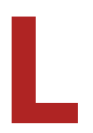

a pneumonie est la plus fréquente cause d'hospitalisation chez les patients qui contractent la maladie à coronavirus 2019 (COVID-19), et beaucoup de ces patients auront besoin d'oxygénothérapie ${ }^{1}$. La pneumonie grave peut entraîner une insuffisance respiratoire hypoxémique aiguë nécessitant une oxygénothérapie ou un soutien respiratoire par ventilation mécanique. L'insuffisance respiratoire aiguë sévère peut entraîner un syndrome de détresse respiratoire aiguë (SDRA), une forme non cardiogénique d'œdème pulmonaire déclenchée par une atteinte directe (p. ex., pneumonie) ou indirecte (p. ex., pancréatite). Le traitement du SDRA repose principalement sur la correction du facteur étiologique et des soins de soutien, ce qui inclut la ventilation mécanique, tout en veillant à prévenir une lésion pulmonaire induite par la ventilation. La ventilation en décubitus ventral est une technique qui est employée et évaluée depuis une trentaine d'années chez des patients ventilés mécaniquement pour des SDRA de diverses intensités; les bienfaits les plus marqués ayant été observés chez ceux qui avaient un SDRA de modéré à grave, pour lesquels on considère désormais qu'il s'agit d'une norme thérapeutique².

Pendant la pandémie de COVID-19, alors que les systèmes de santé peinaient à accommoder une clientèle en hausse atteinte d'insuffisance respiratoire aiguë, des rapports ont émergé au sujet de l'avantage potentiel du décubitus ventral chez les patients atteints de COVID-19 qui étaient hypoxémiques et non intubés ${ }^{1}$. Les conclusions de plusieurs études d'observation ont laissé entendre que le décubitus ventral pouvait améliorer l'oxygénation des patients munis de dispositifs à bas débit (p. ex., canule nasale) et à plus haut débit (p. ex., canule nasale à haut débit) qui n'étaient pas sous ventilation mécanique ${ }^{1,3-7}$.

\section{Encadré $n^{\circ} 1$ : Données utilisées pour la présente revue}

Nous avons procédé à une interrogation du réseau Medline pour tous les articles publiés en langue anglaise entre le $1^{\text {er }}$ et le 14 septembre 2020, à partir des mots ou expressions clés « prone position » ou « prone positioning » dans le contexte du traitement de la maladie à coronavirus 2019 (COVID-19).

\section{POINTS CLÉS}

- Le décubitus ventral a largement été adopté comme pratique standard chez les patients atteints d'un syndrome de détresse respiratoire aiguë sévère ventilés mécaniquement sur la base de données probantes de grande qualité.

- Le décubitus ventral chez les patients présentant une insuffisance respiratoire hypoxémique qui sont éveillés, qui respirent spontanément et qui ne sont pas intubés est possible dans les unités de soins; des données sur son utilisation pour la prise en charge des patients atteints de pneumonie causée par la maladie à coronavirus 2019 (COVID-

19) ont émergé et montrent sa capacité d'améliorer l'oxygénation et de réduire la dyspnée.

- Les études n'ont pas encore fourni aux cliniciens des outils pour prédire quels patients atteints de COVID-19 sont les plus susceptibles de voir leur état s'améliorer avec le décubitus ventral, et elles n'ont pas encore démontré si ce dernier permet de retarder ou d'éviter la ventilation effractive; elles n'ont pas non plus montré un avantage au plan de la mortalité.

- Le décubitus ventral chez le patient ventilé mécaniquement s'accompagne du risque de débranchement de son tube endotrachéal et de ses divers cathéters; ce risque est moindre chez le patient éveillé et non ventilé, tout comme le risque de pression indue chez le patient éveillé qui peut changer de position seul.

- Il faudra réaliser des essais randomisés et contrôlés pour mieux comprendre les effets positifs et négatifs du décubitus ventral chez les patients atteints de COVID-19 qui respirent spontanément.

Nous abordons ici le décubitus ventral, ses mécanismes physiologiques, ses candidats potentiels, les données probantes qui s'accumulent au sujet de son efficacité chez les patients présentant une insuffisance respiratoire hypoxémique liée à une pneumonie causée par la COVID-19 et les inconvénients potentiels de la manœuvre. En résumant les articles disponibles pour guider les médecins dans l'utilisation du décubitus ventral chez cette population (encadré $n^{\circ} 1$ ), nous attirons aussi leur attention sur d'importants secteurs à explorer pour la recherche. 


\section{Qu'est-ce que le décubitus ventral et quelle est son influence sur la fonction pulmonaire?}

Dans le contexte d'un SDRA grave, la ventilation en décubitus dorsal exerce des forces gravitationnelles qui pourraient exacerber l'œdème pulmonaire et l'atélectasie dans les zones pulmonaires dépendantes (postérieures). Les organes abdominaux déplacent le diaphragme postérieur vers le haut, ce qui aggrave le collapsus pulmonaire postérieur ${ }^{8}$. Une vasoconstriction pulmonaire hypoxémique anormale peut aussi contribuer à un déséquilibre du rapport ventilation/perfusion (V/Q) ${ }^{9}$.

Le décubitus ventral consiste à placer le patient face vers le bas sur son thorax antérieur et son abdomen pour profiter des changements physiologiques susceptibles d'améliorer l'oxygénation en réduisant le déséquilibre $V / Q$ et potentiellement, en atténuant l'atteinte pulmonaire. En décubitus ventral, l'expansion de la paroi thoracique antérieure se trouve limitée; cela provoque une compliance thoraco-pulmonaire plus homogène (figure 1) et les forces gravitationnelles exercées sur le parenchyme pulmonaire entraînent un meilleur recrutement des zones postérieures et permettent à une plus grande proportion d'alvéoles de participer aux échanges gazeux. Une distribution plus égale des forces de stress exercées sur les poumons par le diaphragme s'observe aussi en décubitus ventral, ce qui pourrait atténuer l'atteinte pulmonaire, tant durant la ventilation mécanique que lors de la respiration spontanéee ${ }^{8,10}$. La position améliore aussi le mouvement inférieur du diaphragme, qui atténue la compression sur les zones pulmonaires postérieures atélectasiques, augmentant ainsi la recrutabilité pulmonaire ${ }^{10,11}$. Le décubitus ventral exerce toutefois relativement peu d'effet sur la perfusion pulmonaire, car la majeure partie du débit sanguin est orientée vers les zones pulmonaires postérieures, tant en décubitus dorsal qu'en décubitus ventral ${ }^{9}$. Le résultat est une amélioration de l'équilibre $V / Q$, une baisse de la fraction du shunt pulmonaire et une amélioration de l'oxygénation.

\section{Comment s'y prend-on pour placer les patients en décubitus ventral?}

Faire passer un patient intubé et muni de plusieurs tubulures du décubitus dorsal au décubitus ventral est une manœuvre très délicate. Étant donné que la plupart des patients sont lourdement « sédationnés » et habituellement curarisés pour faciliter la ventilation, il faut au moins 3 employés d'expérience pour bien coordonner la manœuvre ${ }^{12}$.

Pendant la pandémie de COVID-19, certains établissements ont tenté le décubitus ventral chez des patients hypoxémiques éveillés et non intubés, soit aux urgences, soit dans des unités ambulatoires. Pour les patients non intubés, plusieurs des risques associés au décubitus ventral sont atténués (p. ex., risque d'extubation). Le processus est plus facile si les patients peuvent se tourner sans aide physique; toutefois, surtout durant la période initiale du décubitus ventral, un membre du personnel doit être présent pour vérifier que le changement de position n'a pas entraîné la déconnexion du tube d'oxygène, des lignes intraveineuses ou de tout autre cathéter (p. ex., sonde Foley). L'oxy-

\section{Décubitus dorsal}

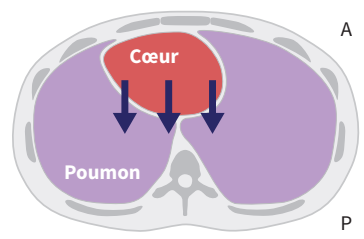

Pression gravitationnelle du cœur et du médiastin sur les poumons.

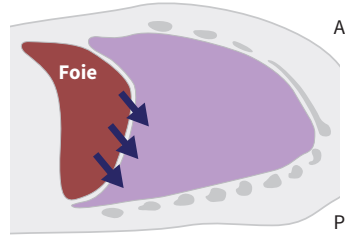

Effets compressifs exercés par les organes abdominaux sur les poumons.

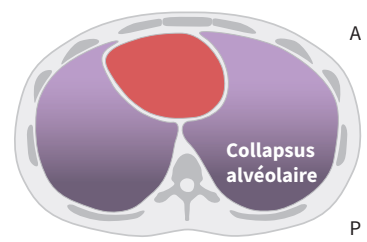

Expansion de la paroi thoracique et compliance globalement moins homogène de la paroi thoracique

\section{Décubitus ventral}

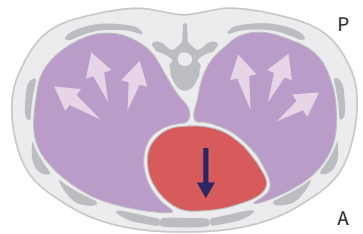

Réduction de la pression gravitationnelle du cœur et du médiastin sur les poumons.

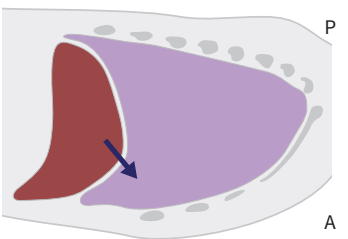

Réduction des effets compressifs exercés par les organes sur les poumons.

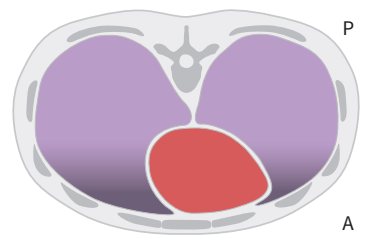

Compliance plus homogène de la paroi thoracique due à une restriction du mouvement de la paroi thoracique antérieure.
Figure 1 : Comparaison de certains effets physiologiques du décubitus dorsal (à gauche) et ventral (à droite). En décubitus ventral, les autres organes exercent moins de pression sur les poumons, ce qui améliore la compliance pulmonaire et le rapport ventilation-perfusion. Panneau du haut : Les flèches bleu foncé indiquent la direction de la pression exercée sur les poumons par le cœur. Panneau du milieu : Les flèches indiquent la direction de la pression exercée par les organes abdominaux sur les poumons. En décubitus ventral, une force moindre est exercée sur les poumons par ces organes, ce qui améliore la compliance pulmonaire en réduisant l'effort nécessaire pour prendre de l'expansion et s'opposer à ces pressions. Panneau du bas : La gradation du gris représente la perfusion pulmonaire; les tons plus sombres représentent un déséquilibre ventilation-perfusion plus marqué dû au collapsus alvéolaire à la région postérieure chez le patient en décubitus dorsal (moins marqué chez le patient en décubitus ventral étant donné que cette position favorise l'expansion thoracique). Remarque : $\mathrm{A}=$ antérieur, $\mathrm{P}=$ postérieur. Illustration créée par Mike Fralick et Saba Manzoor, adaptée par Émilie Lacharité.

métrie de pouls en continu et une surveillance étroite de la fréquence respiratoire sont souhaitables pour s'assurer que l'état clinique du patient ne se détériore pas et pour faciliter la surveillance respiratoire chez les patients inscrits à des études cliniques.

On peut placer des oreillers ou des couvertures roulées de la façon appropriée sous les points de pression, par exemple au niveau du thorax supérieur et du bassin, pour rendre le décubitus 
ventral plus confortable et tolérable et pour réduire la pression intraabdominale qui peut se transférer vers les poumons. Les patients qui respirent spontanément peuvent changer leur tête et leurs bras de position au moins toutes les 2 heures pour éviter les ulcères de pression ${ }^{12}$.

\section{Quels patients sont candidats au décubitus ventral?}

Avant la pandémie de COVID-19, le décubitus ventral servait principalement aux patients atteints d'un SDRA grave qui étaient sous ventilation mécanique ${ }^{13}$. Chez les patients atteints de COVID-19 qui respirent spontanément et qui ne sont pas intubés, les données d'observation suggèrent que le décubitus ventral améliore l'oxygénation chez ceux qui tolèrent cette position. Plusieurs essais randomisés et contrôlés (ERC) sont en cours pour étudier l'efficacité du décubitus ventral chez les patients qui ont besoin d'un supplément d'oxygène à bas débit dans une unité de soins (essais cliniques nos NCT04383613, NCT04402879, NCT04517123) et les patients présentant une insuffisance respiratoire plus grave, qui requièrent de l'oxygène à plus haut débit (essais cliniques nos NCT04350723, NCT04543760).

Le décubitus ventral est à éviter chez les patients qui respirent spontanément, mais qui sont susceptibles de nécessiter rapidement une intubation (p. ex., altération du niveau de conscience, aggravation de l'hypoxie malgré un apport maximal en oxygène) ou chez ceux qui présentent des contre-indications anatomiques décrites dans la littérature reconnue sur le SDRA (p. ex., traumatisme au visage; chirurgie récente à l'abdomen, au thorax ou à la colonne, pose récente d'un stimulateur cardiaque ou fracture instable de la colonne ou du bassin) $)^{1,4,12,14}$.

On en sait peu sur les effets du décubitus ventral sur le fœtus durant la grossesse, étant donné que les patientes enceintes sont souvent exclues des essais (une pratique récemment remise en question durant la pandémie de COVID-1915), même si un rapport de cas a déjà décrit la réussite du décubitus ventral chez une patiente enceinte atteinte d'une grave pneumonie virale ${ }^{16}$. Un protocole et un guide sur le décubitus ventral établi par des experts ont récemment été préparés à l'intention des médecins qui suivent des cas obstétricaux ${ }^{17}$, et l'utilisation réussie du décubitus ventral chez une patiente enceinte atteinte de COVID-19 a récemment été documentée sous forme de rapport de $\operatorname{cas}^{18}$.

\section{Pour qui le décubitus ventral est-il efficace?}

\section{Patients atteints de SDRA}

Le décubitus ventral est évalué depuis les années 1970 pour la prise en charge des patients atteints de SDRA ${ }^{13}$. Chez les patients atteints d'un SDRA de modéré à grave, le décubitus ventral prolongé (au moins à $12 \mathrm{~h} / \mathrm{j}$ ) a effectivement réduit la mortalité et constitue la norme thérapeutique pour leur prise en charge ${ }^{2,13}$. La ventilation en décubitus ventral réduirait l'atteinte pulmonaire induite par le respirateur grâce à une distribution plus uniforme du volume courant, ce qui réduit le stress non physiologique exercé sur les poumons. Un grand ERC multicentrique publié en 2013 et regroupant 474 participants en France a révélé que la ventilation chez les patients atteints de SDRA de modéré à grave (rapport pression partielle de l'oxygène/fraction inspirée en oxygène $\left[\mathrm{PaO}_{2} / \mathrm{FiO}_{2}\right]<150 \mathrm{~mm} \mathrm{Hg}$ ) placés en décubitus ventral pendant 16 heures par jour a été a associée à une amélioration de la mortalité à 28 jours comparativement au décubitus dorsal (risque relatif [RR] 0,39, intervalle de confiance [IC] à $95 \% 0,25-$ $0,63)^{12}$. Une méta-analyse de 8 ERC ayant regroupé les données concernant 2129 patients atteints de SDRA ventilés mécaniquement a par la suite montré que les patients qui avaient un SDRA de modéré à grave assignés aléatoirement au décubitus ventral pendant au moins 12 heures par jour présentaient un taux de mortalité moindre ([RR] 0,74, IC à $95 \% 0,56-0,99)$ comparativement à ceux ventilés en décubitus dorsal ${ }^{13}$.

Le décubitus ventral a été tenté chez des patients atteints de SDRA lié à la COVID-19 et même si on ne s'entend pas sur les possibles attributs physiologiques particuliers du SDRA lié à la COVID-19 ${ }^{19,20}$, certaines lignes directrices (p. ex., Surviving Sepsis Campaign) recommandent d'envisager le décubitus ventral chez les patients atteints de SDRA grave lié à la COVID-19 parce qu'il s'est révélé bénéfique dans le contexte du SDRA grave ${ }^{21-23}$.

\section{Patients non intubés}

Avant la pandémie de COVID-19, le décubitus ventral était peu utilisé pour la prise en charge des patients non intubés présentant une insuffisance respiratoire hypoxémique. Une étude de cohorte rétrospective menée dans un seul centre en 2015 a évalué la réponse de 15 patients ayant fait l'objet en tout de 43 manœuvres de décubitus ventral. La plupart des participants ( $n=13)$ avaient un diagnostic de pneumonie et lors de $42 \%$ des manœuvres, une ventilation non effractive était utilisée. L'hypoxémie des participants s'est significativement améliorée en décubitus ventral $\left(\mathrm{PaO}_{2} / \mathrm{FiO}_{2}\right.$ moyenne en décubitus dorsal 127 [écart-type (É.-T.) 49] mm Hg, $\mathrm{PaO}_{2} / \mathrm{FiO}_{2}$ moyenne en décubitus ventral 186 [É.-T. 72] mm Hg; $p<0,05$ ), même si l'amélioration n'a pas semblé persister lorsque les patients revenaient en décubitus dorsal?.

Une étude de cohorte prospective multicentrique s'est penchée sur l'effet d'une combinaison décubitus ventral et soit ventilation non effractive, soit canule nasale à haut débit chez 20 participants éveillés, dont 11 souffraient de pneumonie virale ${ }^{4}$. Une approche séquentielle a été utilisée pour ajouter le décubitus ventral soit à un système d'administration de l'oxygène basé sur la réponse clinique du patient, soit à une alternance entre ventilation non effractive et canule nasale à haut débit. L'hypoxémie des patients au départ n'a pas été rapportée, même si tous les patients répondaient aux critères de SDRA. Les auteurs ont noté des améliorations de l'oxygénation quand le décubitus ventral était ajouté à la canule nasale à haut débit, mais non à la ventilation non effractive et, même si 11 participants ont évité l'intubation, l'étude a révélé une augmentation non significative chez les patients atteints de SDRA grave ayant nécessité une intubation et une ventilation mécanique ${ }^{4}$.

La littérature servant à orienter l'utilisation du décubitus ventral chez les patients atteints d'insuffisance respiratoire liée à la COVID19 non intubés qui respirent spontanément comprend des rapports de cas, des séries de cas et des études d'observation ${ }^{1,3,4,6,7,14}$. 
Tableau 1 : Sommaire des données probantes à l'appui du décubitus ventral chez les patients atteints de maladie à coronavirus 2019 non intubés

\begin{tabular}{|c|c|c|c|c|c|c|c|}
\hline Étude & Modèle d'étude & $\begin{array}{c}\text { Population } \\
\text { étudiée }\end{array}$ & $\begin{array}{l}\text { Principaux } \\
\text { paramètres }\end{array}$ & Lieu & Oxygénothérapie & $\begin{array}{c}\text { Protocole de } \\
\text { décubitus ventral }\end{array}$ & Principales conclusions \\
\hline $\begin{array}{l}\text { Caputo } \\
\text { et al. }{ }^{6}\end{array}$ & $\begin{array}{l}\text { Étude de cohorte } \\
\text { prospective menée } \\
\text { dans un seul centre }\end{array}$ & $\begin{array}{l}n=50 \\
\mathrm{SpO}_{2} \text { au départ } \\
\text { avant dv: } \\
\text { médiane } 84 \% \\
\text { (ÉIQ } 75 \%-90 \% \text { ) }\end{array}$ & $\begin{array}{l}\text { Changement de } \\
\text { l'oxygénation }\left(\mathrm{SpO}_{2}\right) \text {, } \\
\text { taux d'intubation } \\
\text { dans les } 24 \text { premières } \\
\text { heures suivant } \\
\text { l'arrivée aux urgences }\end{array}$ & Urgence & $\begin{array}{l}\text { MSR ( } 38 \text { patients sur } \\
50,76 \% \text { ) ou CNHD } \\
\text { ( } 12 \text { patients sur } 50 \text {, } \\
24 \% \text { ) }\end{array}$ & $\begin{array}{l}5 \text { minutes de DV et } \\
\text { oxygénothérapie } \\
\text { inchangée }\end{array}$ & $\begin{array}{l}\text { - } \mathrm{SpO}_{2} \text { médiane après un } \\
\text { seul épisode de } 5 \text { min. de } \\
\text { DV: } 94 \% \text { (ÉlQ } 90 \%-95 \% \text { ) } \\
\text { - } 18 \text { patients sur } 50 \text { (36\%) } \\
\text { intubés dans les } 72 \mathrm{~h} \\
\text { suivant leur arrivée }\end{array}$ \\
\hline $\begin{array}{l}\text { Elharrar } \\
\text { et al. }{ }^{1}\end{array}$ & $\begin{array}{l}\text { Étude prospective } \\
\text { avant/après menée } \\
\text { dans un seul centre }\end{array}$ & $\begin{array}{l}n=24 \\
\mathrm{PaO}_{2} \text { moyenne au } \\
\text { départ : } 72,8 \text { (É.-T. } \\
\text { 14,2) } \mathrm{mm} \mathrm{Hg}\end{array}$ & $\begin{array}{l}\text { Augmentation de la } \\
\mathrm{PaO}_{2} \geq 20 \% \text { c. départ }\end{array}$ & $\begin{array}{l}\text { Unité de } \\
\text { médecine }\end{array}$ & $\begin{array}{l}16 \text { patients sur } 24 \\
(67 \%) \text { avec } \mathrm{CN}<4 \mathrm{~L} / \\
\text { min; } 8 \text { patients sur } 25 \\
(33 \%) \text { avec } \mathrm{CN} \geq 4 \mathrm{~L} / \\
\text { min ou } \mathrm{CNHD}\end{array}$ & $\begin{array}{l}\text { Épisode simple de } \\
\text { DV; durée selon le } \\
\text { confort }\end{array}$ & $\begin{array}{l}\text { - } 6 \text { patients sur } 24(25 \%) \\
\text { présentaient le } \\
\text { paramètre principal } \\
\text { - } \text { Aucune réponse durable } \\
\text { au DV après retour au } \\
\text { décubitus dorsal } \\
\text { - } 15 \text { patients sur } 24 \text { ( } 63 \%) \\
\text { ont toléré le DV pendant } \\
>3 \mathrm{~h}\end{array}$ \\
\hline $\begin{array}{l}\text { Sartini et } \\
\text { al. }^{3}\end{array}$ & $\begin{array}{l}\text { Étude transversale } \\
\text { menée dans un seul } \\
\text { centre }\end{array}$ & $\begin{array}{l}n=15 \\
\mathrm{PaO}_{2} / \mathrm{FiO}_{2} \\
\text { moyenne } \\
\text { au départ : } 157 \\
\text { (É.-T. 43) mm Hg }\end{array}$ & $\begin{array}{l}\text { Changement de } \\
\text { l'oxygénation et des } \\
\text { signes vitaux } \\
\text { respiratoires }\end{array}$ & $\begin{array}{l}\text { Unité de } \\
\text { médecine }\end{array}$ & VNE & $\begin{array}{l}\text { Nombre médian de } \\
\text { cycles de DV : } 2 \text { (ÉIQ } \\
\text { 1-3), durée } \\
\text { médiane : } 3 \text { h (ÉlQ } \\
\text { 1-6) }\end{array}$ & $\begin{array}{l}\text { - Tous les patients ont eu } \\
\text { une amélioration de leurs } \\
\mathrm{FR} \text { et } \mathrm{PaO}_{2} / \mathrm{FiO}_{2} \text { en } \mathrm{DV}^{\star} \\
\text { - } 12 \text { patients sur } 15 \text { ( } 80 \%) \\
\text { ont eu une amélioration } \\
\text { de leur oxygénation } \\
\text { après le } \mathrm{DV}^{\star} \\
\text { - } 11 \text { patients sur } 15(73 \%) \\
\text { ont eu une amélioration } \\
\text { de leur confort durant } \\
\text { le DV }\end{array}$ \\
\hline $\begin{array}{l}\text { Coppo et } \\
\text { al. }^{24}\end{array}$ & $\begin{array}{l}\text { Étude de cohorte } \\
\text { prospective menée } \\
\text { dans un seul centre }\end{array}$ & $\begin{array}{l}n=56 \\
\mathrm{PaO}_{2} / \mathrm{FiO}_{2} \\
\text { moyenne au } \\
\text { départ : } 180,5 \\
\text { (É.-T. } 76,6) \mathrm{mm} \mathrm{Hg}\end{array}$ & $\begin{array}{l}\text { Changement de la } \\
\mathrm{PaO}_{2} / \mathrm{FiO}_{2} \text { en } \\
\text { décubitus dorsal } \\
\text { après l'épisode de DV }\end{array}$ & $\begin{array}{l}\text { Unité de } \\
\text { médecine, } \\
\text { Urgence, } \\
\text { ÉSII }\end{array}$ & $\begin{array}{l}\text { CPAP ( } 44 \text { patients } \\
\text { sur } 56,79 \%) \text {, MSR } \\
\text { ( } 9 \text { patients sur } 56, \\
16 \%) \text {, masque } \\
\text { Venturi ( } 3 \text { patients } \\
\text { sur } 56,5 \%)\end{array}$ & $\begin{array}{l}\text { Minimum } 3 \mathrm{~h} \text {, } \\
\text { jusqu'à } 8 \mathrm{~h} \text { selon le } \\
\text { confort; } 25 \text { patients } \\
\text { maintenus }>3 \mathrm{~h}\end{array}$ & $\begin{array}{l}\text { - Amélioration de } \\
\text { l'oxygénation en DV : } \\
\mathrm{PaO}_{2} / \mathrm{FiO}_{2} \text { moyenne } \\
285,5(\text { É-T. 112,9) } \\
\text { - Amélioration non } \\
\text { significative de } \\
\text { l'oxygénation après DV } \\
\text { chez } 50 \% \text { des } \\
\text { participants }\end{array}$ \\
\hline $\begin{array}{l}\text { Winearls } \\
\text { et al. }{ }^{25}\end{array}$ & $\begin{array}{l}\text { Étude de cohorte } \\
\text { rétrospective } \\
\text { menée dans un seul } \\
\text { centre }\end{array}$ & $\begin{array}{l}n=24 \\
\mathrm{PaO}_{2} / \mathrm{FiO}_{2} \\
\text { moyenne au } \\
\text { départ sous C-PAP: } \\
143 \text { (É.-T. 73) mm } \\
\mathrm{Hg}\end{array}$ & $\begin{array}{l}\text { Changement des } \\
\text { signes vitaux } \\
\text { respiratoires, } \\
\text { tolérabilité et durée } \\
\text { du DV }\end{array}$ & SII & CPAP & $\begin{array}{l}\text { DV débuté après une } \\
\text { durée médiane de } \\
30 \text { h (ÉIQ 7-99) de } \\
\text { CPAP; } \\
\text { durée moyenne du } \\
\text { DV au cours des } 24 \\
\text { premières heures: } \\
8 \text { h (É.-T. 5), maintenu } \\
\text { pendant } 10 \text { j (É.-T. 5) }\end{array}$ & $\begin{array}{l}\text { Augmentation } \\
\text { moyenne significative } \\
\text { de la } \mathrm{PaO}_{2} / \mathrm{FiO}_{2} \text { avec le } \\
\text { DV et le CPAP : } 252 \text { (É.- } \\
\mathrm{T} .87 \text { ) mm } \mathrm{Hg}, p<0,01 ; \\
\text { maintenue } 1 \mathrm{~h} \text { après } \\
\text { l'arrêt du DV } \\
\text { - } 2 \text { patients incapables de } \\
\text { tolérer le DV en raison de } \\
\text { l'inconfort }\end{array}$ \\
\hline $\begin{array}{l}\text { Solverson } \\
\text { et al. }{ }^{26}\end{array}$ & $\begin{array}{l}\text { Étude de cohorte } \\
\text { rétrospective } \\
\text { multicentrique }\end{array}$ & $\begin{array}{l}n=17 \\
\mathrm{PaO}_{2} / \mathrm{FiO}_{2} \\
\text { médiane au } \\
\text { départ : } 152 \text { (ÉIQ } \\
\text { 97-233) mm Hg }\end{array}$ & $\begin{array}{l}\text { Tolérabilité du DV, } \\
\text { paramètres } \\
\text { physiologiques et } \\
\text { cliniques }\end{array}$ & $\begin{array}{l}\text { Unité de } \\
\text { médecine, } \\
\text { USI }\end{array}$ & $\mathrm{CN}, \mathrm{MSR}, \mathrm{CNHD}$ & $\begin{array}{l}\text { Nombre médian de } \\
\text { séances de DV/j } 2 \\
\text { (ÉlQ 1-6) et durée } \\
\text { médiane de la } \\
\text { première séance } \\
75 \text { min (ÉIQ 30-480) }\end{array}$ & $\begin{array}{l}\text { - Ralentissement de la FR } \\
\text { et amélioration de } \\
\text { l'oxygénation en DV } \\
\text { - } 35 \%(n=6) \text { des } \\
\text { patients ont cessé le DV } \\
\text { après } \leq 60 \text { minutes en } \\
\text { raison de douleurs } \\
\text { musculosquelettiques } \\
\text { ou d'inconfort général }\end{array}$ \\
\hline $\begin{array}{l}\text { Ferrando } \\
\text { et al. }{ }^{27}\end{array}$ & $\begin{array}{l}\text { Étude de cohorte } \\
\text { prospective } \\
\text { multicentrique }\end{array}$ & $\begin{array}{l}n=199 ; 144 \\
\text { patients n'ont eu } \\
\text { que la CNHD et } 55 \\
\text { ont eu la CNHD + } \\
\text { DV }\end{array}$ & $\begin{array}{l}\text { Paramètres } \\
\text { physiologiques et } \\
\text { cliniques, incluant le } \\
\text { risque d'intubation }\end{array}$ & USI & CNHD & $\begin{array}{l}\text { On tenait compte de } \\
\text { la DV que si elle } \\
\text { durait }>16 \mathrm{~h} / \mathrm{j} \text {, } \\
\text { indépendamment } \\
\text { du nombre de } \\
\text { séances }\end{array}$ & $\begin{array}{l}\text { - Augmentation } \\
\text { significative de la } \mathrm{PaO}_{2} / \\
\mathrm{FiO}_{2} \text { avec NCHD + DV } \\
\text { - Tendance à un report de } \\
\text { l'intubation chez les } \\
\text { patients sous CNHD + DV; } \\
\text { aucune différence dans } \\
\text { les taux d'intubation } \\
\text { entre les groupes }\end{array}$ \\
\hline
\end{tabular}

Remarque : $\mathrm{CN}=$ canule nasale, $\mathrm{CNHD}=$ canule nasale à haut débit, $\mathrm{CPAP}=$ unité de pneumologie placés sous ventilation à pression positive continue, $\mathrm{DV}=$ décubitus ventral, ÉlQ = éventail interquartile, $\mathrm{E}$.- $\mathrm{T}$. = écart-type, $\mathrm{FiO}_{2}=$ fraction inspirée en oxygène, $\mathrm{FR}=$ fréquence respiratoire, $\mathrm{MSR}=$ masque à oxygène sans réinspiration, $\mathrm{PaO} \mathrm{O}_{2}=$ pression partielle d'oxygène, $\mathrm{SII}=$ soins intensifs intermédiaires, $\mathrm{SpO}_{2}=$ saturation en oxygène de l'hémoglobine, USI = unité de soins intensifs, VNE = ventilation non effractive.

*Données précises non disponibles. 
Le nombre important de patients atteints de COVID-19 à l'échelle mondiale a permis l'évaluation du décubitus ventral à l'extérieur des unités de soins intensifs (USI) : urgences, unités médicales et unités chirurgicales reconverties.

Sur la base des données observationnelles disponibles (résumées au tableau 1), le décubitus ventral chez cette population de patients semble améliorer l'oxygénation de nombreux patients ${ }^{1,3,6,7,28-32}$. Par exemple, une étude prospective non randomisée regroupant 50 patients placés en décubitus ventral aux urgences a montré une amélioration de l'oxygénation en l'espace de 5 minutes même si $36 \%$ ont eu besoin d'une intubation en l'espace d'environ 72 heures $^{6}$. La ventilation non effractive et le décubitus ventral ont été utilisés concomitamment dans une petite étude transversale sur 15 participants atteints de COVID-19 et ont amélioré l'oxygénation, y compris chez $80 \%$ des participants qui ont connu une amélioration après être revenus en décubitus dorsal ${ }^{3}$. Une étude de cohorte rétrospective a passé en revue les paramètres de 24 patients d'une unité de pneumologie placés sous ventilation à pression positive continue (CPAP) en conjonction avec le décubitus ventral, et a noté que même si l'ajout du CPAP n'a pas amélioré significativement la saturation artérielle en oxygène, la combinaison CPAP et décubitus ventral y est arrivée (saturation moyenne en oxygène au départ 94\% (É.-T. 3\%) et postdécubitus ventral $96 \%$ (écart-type $2 \% ; p<0,05)^{25}$. Cette amélioration se maintenait 1 heure après le retour des participants au décubitus dorsal ${ }^{25}$. Une étude de cohorte prospective regroupant 56 patients qu'on avait placés en décubitus ventral soit à l'urgence, soit à l'unité de médecine ou l'unité d'observation ${ }^{24} \mathrm{a}$ révélé que le décubitus ventral était faisable chez $84 \%$ des participants et améliorait significativement l'oxygénation, même si cela ne persistait pas lorsque les patients revenaient en décubitus dorsal. Dans une petite étude prospective menée dans un seul centre en France, l'utilisation d'un seul épisode de décubitus ventral a été bien tolérée, mais n'a amélioré l'oxygénation que chez $25 \%$ des participants, la moitié de ceux qui ont répondu ayant connu une amélioration persistante ${ }^{1}$. Toutefois, l'absence de randomisation dans ces études signifie que les bienfaits observés pourraient être dus au décubitus ventral, à un biais de sélection ou à un facteur de confusion lié à l'indication.

Les données selon lesquelles le décubitus ventral réduit le recours à l'intubation sont manquantes. Certaines études d'observation ont montré que le décubitus ventral ralentit la fréquence respiratoire ${ }^{3,28}$, ce qui pourrait réduire le risque de lésions pulmonaires auto-infligées chez les patients ${ }^{3,10}$, même si l'extrapolation à partir de ce paramètre-substitut doit se faire avec prudence ${ }^{1,3,6,7}$. Chez les patients atteints d'un SDRA de léger à modéré qui ont été intubés ou qui ont été placés pendant de courtes périodes $(<12 \mathrm{~h} / \mathrm{j})$ en décubitus ventral, l'amélioration de l'oxygénation n'a pas été en corrélation avec un avantage au plan de la mortalité ${ }^{13}$. En outre, les données sur la persistance de l'amélioration de l'oxygénation une fois les patients respirant spontanément revenus en décubitus dorsal ne sont cohérentes ${ }^{1,3,6,11,24,25,28}$; on en déduit que des ERC portant sur les paramètres cliniques chez des patients atteints de COVID-19 placés en décubitus ventral s'imposent. Malgré ces données lacunaires, l'Intensive Care Society du Royaume-Uni. a publié des directives basées sur l'opinion d'experts; elles encouragent l'utilisation du décubitus ventral chez les patients non intubés en raison de sa facilité d'application et de ses bienfaits potentiels ${ }^{33}$.

\section{Quels sont les effets négatifs potentiels du décubitus ventral?}

Chez les patients présentant un SDRA ventilés mécaniquement, les effets négatifs potentiels du décubitus ventral surviennent surtout au moment de la manœuvre pour les installer en décubitus ventral (en raison des risques de débranchement des tubes et cathéters) ou sous forme de séquelles d'une immobilité prolongée chez les patients qui sont incapables de bouger (incluant ulcères de pression, neurapraxie et œdème facial) ${ }^{13}$. En majorité, ces risques sont substantiellement moindres chez les patients qui respirent spontanément et ne sont pas intubés parce qu'ils sont capables de changer de position pour leur confort.

Même si l'inconfort des patients peut limiter le recours au décubitus ventral ou en abréger l'utilisation, un positionnement adéquat et l'utilisation de coussins au niveau des points d'appui le rendent plus tolérable ${ }^{1,4,7,11,31}$. Une étude de cohorte prospective canadienne regroupant 4 hôpitaux de Calgary a évalué l'innocuité et la tolérabilité du décubitus ventral chez des patients non intubés dans des unités de médecine et des USI ${ }^{26}$. L'étude a fait état de 17 participants ayant reçu un nombre médian de 2 séances de décubitus ventral (entre 1 et 6) d'une durée médiane de 75 minutes (entre 30 et 480). Le temps passé en décubitus ventral a été limité par des douleurs au dos ou à l'épaule $(n=2,12 \%)$, un inconfort général $(n=6,35 \%)$ et le délire $(n=1,6 \%)$. Huit patients $(47 \%)$ n'ont éprouvé aucun problème au plan de la tolérabilité. Aucun autre effet indésirable grave n'est survenu ${ }^{26}$.

Il est important de vérifier si le décubitus ventral prolongé chez les patients atteints de COVID-19 éveillés et non intubés accroît le risque de thromboembolie veineuse parce que des études d'observation ont montré que la COVID-19 est associée à un risque accru de thrombose veineuse ${ }^{34}$. Les études publiées sur le décubitus ventral chez des patients non intubés ont peu mentionné ces effets négatifs.

Si le décubitus ventral retarde plutôt que de prévenir l'intubation, il pourrait accroître les taux d'intubation urgente, qui comporte ses propres risques. Une étude de cohorte multicentrique de 2020 regroupant 36 hôpitaux en Espagne et à Andorre a révélé que l'utilisation du décubitus ventral avec une canule nasale à haut débit n'a pas réduit le risque d'intubation (RR 1,002, IC à 95\% 0,531-1,890; $p=0,99)^{27}$. Cette étude a aussi montré une tendance non significative à l'allongement de l'intervalle avant l'intubation (2,0 c. 4,1 j, $p=0,054)$, ce qui fait craindre d'un préjudice potentiel dû au retard de l'intubation ${ }^{27}$.

\section{Quelles questions restent en suspens?}

Aucune étude n'a encore fourni aux médecins des outils leur permettant de prédire quels patients sont les plus susceptibles de voir leur état s'améliorer; n'a caractérisé les bienfaits relatifs du décubitus ventral, de la canule nasale à haut débit et de la ventilation non effractive (seuls et ensemble); n'a déterminé la « dose » optimale de 
décubitus ventral; ni, ce qui est plus déterminant, n'a prouvé que le décubitus ventral permet de retarder ou d'éviter le recours à la ventilation effractive. On ignore si le décubitus ventral peut réduire les coûts de santé parce que les études sur son rapport coût-efficacité sont inexistantes. Même s'il semble que le décubitus ventral puisse être utilisé à l'extérieur des soins intensifs à un coût minime, il pourrait être associé à une plus grande utilisation d'équipement de protection individuelle (EPI) quand plusieurs travailleurs de la santé doivent participer à la manœuvre. Toutefois, si de futures études confirment que l'intervention réduit les admissions aux soins intensifs, cela pourrait se traduire par des économies substantielles. Même si la ventilation mécanique est retardée ou évitée, cela n'entraînera pas forcément une amélioration des résultats chez les patients; il sera donc crucial d'identifier les patients non intubés qui sont peu susceptibles de bénéficier d'un essai du décubitus ventral. En outre, il faudra mener des ERC rigoureux pour répondre à ces questions.

\section{Quels sont les enjeux logistiques de l'utilisation du décubitus ventral durant la pandémie de COVID-19?}

L'utilisation du décubitus ventral chez les patients atteints de COVID-19 hospitalisés dans des unités de médecine pourrait se répandre afin de prévenir la ventilation mécanique si les ressources des soins intensifs sont trop sollicitées. Comme les études de modélisation ont évoqué une telle possibilité pour les hôpitaux canadiens si les mesures demandées par la santé publique ne sont pas respectées ${ }^{35,36}$, les hôpitaux devraient par conséquent préparer ou adopter des lignes de conduite concernant le décubitus ventral, promouvoir un transfert rapide des connaissances et la formation du personnel clinique. À titre d'exemple, Doussot et ses collègues ont décrit la création d'une équipe spécialisée pour le décubitus ventral dans un hôpital régional en France ${ }^{37}$. Chirurgiens, personnel infirmier, physiothérapeutes et autres professionnels de la santé ont accepté de recevoir la formation, qui incluait une liste de vérification, une simulation et une formation sur l'utilisation appropriée de l'EPI ${ }^{37}$.

\section{Conclusion}

Le décubitus ventral a largement été adopté dans la pratique standard pour les patients atteints de SDRA grave ventilés mécaniquement sur la base de solides données probantes tirées d'ERC. Or, depuis que la pandémie de COVID-19 a surchargé certains systèmes de santé dans le monde et épuisé les ressources des unités de soins intensifs, le décubitus ventral chez les patients non intubés qui respirent spontanément est devenu une intervention attrayante parce qu'il est facile à mettre en œuvre dans différents contextes de soins et qu'il repose sur des principes physiologiques éprouvés. Même si les données probantes actuelles appuyant le recours au décubitus ventral sont de faible qualité, plusieurs ERC sont en cours et devraient répondre aux questions concernant ses bienfaits cliniques, son profil d'innocuité et son rapport coût-efficacité. Nous avons besoin de solides données probantes pour guider la sélection des patients et le moment du début et de l'arrêt du décubitus ventral.

\section{Références}

1. Elharrar X, Trigui Y, Dols A-M, et al. Use of prone positioning in nonintubated patients with COVID-19 and hypoxemic acute respiratory failure. JAMA 2020; 323:2336-8.

2. Fan E, Del Sorbo L, Goligher EC, et al.; American Thoracic Society; European Society of Intensive Care Medicine; Society of Critical Care Medicine. An official American Thoracic Society/European Society of Intensive Care Medicine/Society of Critical Care Medicine clinical practice guideline: mechanical ventilation in adult patients with acute respiratory distress syndrome [Erratum publié dans Am J Respir Crit Care Med 2017;195:1540]. Am J Respir Crit Care Med 2017;195: 1253-63.

3. Sartini C, Tresoldi M, Scarpellini $P$, et al. Respiratory parameters in patients with COVID-19 after using noninvasive ventilation in the prone position outside the intensive care unit. JAMA 2020;323:2338-40.

4. Ding L, Wang L, Ma W, et al. Efficacy and safety of early prone positioning combined with HFNC or NIV in moderate to severe ARDS: a multi-center prospective cohort study. Crit Care 2020;24:28.

5. Sun Q, Qiu H, Huang M, et al. Lower mortality of COVID-19 by early recognition and intervention: experience from Jiangsu Province. Ann Intensive Care 2020; 10:33.

6. Caputo ND, Strayer RJ, Levitan R. Early self-proning in awake, non-intubated patients in the emergency department: a single ED's experience during the COVID-19 pandemic. Acad Emerg Med 2020;27:375-8.

7. Scaravilli V, Grasselli G, Castagna L, et al. Prone positioning improves oxygenation in spontaneously breathing nonintubated patients with hypoxemic acute respiratory failure: a retrospective study. J Crit Care 2015;30:1390-4.

8. Scholten EL, Beitler JR, Prisk GK, et al. Treatment of ARDS with prone positioning. Chest 2017;151:215-24.

9. Pappert D, Rossaint R, Slama K, et al. Influence of positioning on ventilationperfusion relationships in severe adult respiratory distress syndrome. Chest 1994;106:1511-6.

10. Telias I, Katira BH, Brochard L. Is the prone position helpful during spontaneous breathing in patients with COVID-19? JAMA 2020;323:2265-7.

11. Slessarev M, Cheng J, Ondrejicka M, et al. Patient self-proning with high-flow nasal cannula improves oxygenation in COVID-19 pneumonia. Can J Anaesth 2020;67:1288-90.

12. Guérin C, Reignier J, Richard J-C, et al.; PROSEVA Study Group. Prone positioning in severe acute respiratory distress syndrome. N Engl J Med 2013;368: 2159-68.

13. Munshi L, Sorbo LD, Adhikari NKJ, et al. Prone position for acute respiratory distress syndrome. A systematic review and meta-analysis. Ann Am Thorac Soc 2017;14(Suppl 4):S280-8.

14. Pérez-Nieto OR, Guerrero-Gutiérrez MA, Deloya-Tomas E, et al. Prone positioning combined with high-flow nasal cannula in severe noninfectious ARDS. Crit Care 2020;24:114.

15. Malinowski AK, Snelgrove J, Okun N. Excluding pregnancy from COVID-19 trials: Protection from harm or the harm of protection? CMAJ 2020;192:E634.

16. Samanta S, Samanta S, Wig J, et al. How safe is the prone position in acute respiratory distress syndrome at late pregnancy? Am J Emerg Med 2014;32:687. e1-3.

17. Tolcher MC, McKinney JR, Eppes CS, et al. Prone positioning for pregnant women with hypoxemia due to coronavirus disease 2019 (COVID-19). Obstet Gynecol 2020;136:259-61.

18. Schnettler WT, Al Ahwel Y, Suhag A. Severe acute respiratory distress syndrome in coronavirus disease 2019-infected pregnancy: obstetric and intensive care considerations. Am J Obstet Gynecol MFM 2020;2:100120.

19. Gattinoni L, Coppola S, Cressoni M, et al. COVID-19 does not lead to a "typical" acute respiratory distress syndrome. Am J Respir Crit Care Med 2020;201: 1299-300.

20. Gattinoni L, Chiumello D, Caironi P, et al. COVID-19 pneumonia: Different respiratory treatments for different phenotypes? Intensive Care Med 2020;46: 1099-102.

21. Ziehr DR, Alladina J, Petri CR, et al. Respiratory pathophysiology of mechanically ventilated patients with COVID-19: a cohort study. Am J Respir Crit Care Med 2020;201:1560-4. 
22. Ferrando C, Suarez-Sipmann F, Mellado-Artigas R, et al.; COVID-19 Spanish ICU Network. Clinical features, ventilatory management, and outcome of ARDS caused by COVID-19 are similar to other causes of ARDS. Intensive Care Med 2020 July 29 [Cyberpublication avant impression]. doi: 10.1007/s00134-020-06192-2.

23. Alhazzani W, Møller MH, Arabi YM, et al. Surviving Sepsis Campaign: guidelines on the management of critically ill adults with coronavirus disease 2019 (COVID-19). Intensive Care Med 2020;46:854-87.

24. Coppo A, Bellani G, Winterton D, et al. Feasibility and physiological effects of prone positioning in non-intubated patients with acute respiratory failure due to COVID19 (PRON-COVID): a prospective cohort study. Lancet Respir Med 2020;8:765-74.

25. Winearls S, Swingwood EL, Hardaker CL, et al. Early conscious prone positioning in patients with COVID-19 receiving continuous positive airway pressure: a retrospective analysis. BMJ Open Respir Res 2020;7:e000711.

26. Solverson K, Weatherald J, Parhar KKS. Tolerability and safety of awake prone positioning COVID-19 patients with severe hypoxemic respiratory failure. Can J Anaesth 2020 Aug. 14 [Cyberpublication avant impression]. doi: 10.1007/ s12630-020-01787-1.

27. Ferrando C, Mellado-Artigas R, Gea A, et al.; COVID-19 Spanish ICU Network. Awake prone positioning does not reduce the risk of intubation in COVID-19 treated with high-flow nasal oxygen therapy: a multicenter, adjusted cohort study. Crit Care 2020;24:597.

28. Zang X, Wang Q, Zhou H, et al. Efficacy of early prone position for COVID-19 patients with severe hypoxia: a single-center prospective cohort study. Intensive Care Med 2020;46:1927-9.

29. Taboada M, Rodríguez N, Riveiro V, et al. Prone positioning in awake non-ICU patients with ARDS caused by COVID-19. Anaesth Crit Care Pain Med 2020;39:581-3.
30. Taboada M, Rodríguez N, Riveiro V, et al. Short-term outcomes of 50 patients with acute respiratory distress by COVID-19 where prone positioning was used outside the ICU. J Clin Anesth 2020;67:110028.

31. Xu Q, Wang T, Qin X, et al. Early awake prone position combined with highflow nasal oxygen therapy in severe COVID-19: a case series. Crit Care 2020; $24: 250$.

32. Thompson AE, Ranard BL, Wei $\mathrm{Y}$, et al. Prone positioning in awake, nonintubated patients with COVID-19 hypoxemic respiratory failure. JAMA Intern Med 2020 June 17 [Cyberpublivation avant impression] doi: 10.1001/jamainternmed .2020 .3030 .

33. Bamford P, Bentley A, Dean J, et al. ICS guidance for prone positioning of the conscious COVID patient 2020. London (UK): Intensive Care Society; 2020. Accessible ici : https://emcrit.org/wp-content/uploads/2020/04/2020-04-12 -Guidance-for-conscious-proning.pdf (consulté le 12 juillet 2020).

34. Klok FA, Kruip MJHA, van der Meer NJM, et al. Incidence of thrombotic complications in critically ill ICU patients with COVID-19. Thromb Res 2020;191:145-7.

35. Tuite AR, Fisman DN, Greer AL. Mathematical modelling of COVID-19 transmission and mitigation strategies in the population of Ontario, Canada. CMAJ 2020;192:E497-505.

36. Barrett K, Khan YA, Mac S, et al. Estimation of COVID-19-induced depletion of hospital resources in Ontario, Canada. CMAJ 2020;192:E640-6.

37. Doussot A, Ciceron F, Cerutti E, et al. Prone positioning for severe acute respiratory distress syndrome in COVID-19 patients by a dedicated team: a safe and pragmatic reallocation of medical and surgical work force in response to the outbreak. Ann Surg 2020 July 24 [Cyberpublication avant impression]. doi: $10.1097 /$ SLA.0000000000004265.
Intérêts concurrents : Michael Fralick est co-investigateur principal de l'étude COVID-PRONE (ClinicalTrials.gov no NCT04383613). Il est également consultant pour Pine Trees Health, une entreprise en démarrage qui développe un test diagnostique basé sur la technique CRISPR pour la maladie à coronavirus 2019. Kevin Venus et Laveena Munshi sont membres du comité de direction de l'étude COVID-PRONE. Aucun autre intérêt concurrent déclaré.

Cet article a été révisé par des pairs.

Affiliations : Département de médecine (Venus, Munshi, Fralick), Université de Toronto; Division de médecine interne générale et de gériatrie (Venus), Réseau universitaire de santé; Division interdépartementale de médecine intensive (Munshi) et Division de médecine interne générale et de gériatrie (Fralick), Système de santé Sinaï, Toronto, Ontario.

Collaborateurs : Tous les auteurs ont contribué à l'élaboration et à la conception des travaux. Kevin Venus et Michael Fralick ont contribué à l'acquisition, à l'analyse et à l'interprétation des données. Tous les auteurs ont participé à la rédaction du manuscrit, en ont révisé de façon critique le contenu intellectuel important, ont donné leur approbation finale pour la version destinée à être publiée, et assument l'entière responsabilité de tous les aspects du travail.

Financement : Aucun financement externe n'a été reçu pour ces travaux.

Propriété intellectuelle du contenu : Il s'agit d'un article en libre accès distribué conformément aux modalités de la licence Creative Commons Attribution (CC BY-NC-ND 4.0), qui permet l'utilisation, la diffusion et la reproduction dans tout médium à la condition que la publication originale soit adéquatement citée, que l'utilisation se fasse à des fins non commerciales (c.-à-d., recherche ou éducation) et qu'aucune modification ni adaptation n'y soit apportée. Voir : https://creativecommons. org/licenses/by-nc-nd/4.0/

Remerciements : Les auteurs remercient Saba Manzoor qui a aidé à créer la figure 1.

Correspondance : Michael Fralick, mike.fralick@mail.utoronto.ca 